\title{
Restricting the fourth reading*
}

\author{
Ido Benbaji \\ MIT
}

\begin{abstract}
Szabó (2010, 2011) argues that DPs in intensional contexts have specificopaque readings, in which their determiner scopes above some intensional operator while their restrictor is nevertheless interpreted in the scope of the operator. This "fourth reading" poses a potential problem to prominent theories of intensionality (e.g. Percus 2000, Keshet 2008) in which wide quantificational scope (specificity) implies transparency. We attempt to restrict the scope of the problem by demonstrating that an important sub-class of Szabó's examples is syntactically restricted to relativization environments, and can be generated by invoking independently motivated mechanisms for NP reconstruction into relative clauses without calling into question commonly made assumptions about intensional constructions. The analysis correctly predicts fourth readings to be unavailable when a raising structure is independently blocked.
\end{abstract}

Keywords: bare quantification, fourth reading, reconstruction, relative clause

\section{Introduction}

DPs in intensional contexts can differ both in the worlds in which their restrictor is evaluated, and in whether their determiner scopes above or below the intensionalityintroducing operator. When the restrictor is evaluated in the worlds introduced by the intensional operator, we say that it receives an opaque interpretation, as opposed to the transparent interpretation obtained when it is not. When the determiner outscopes the intensional operator, individuating a specific entity when it is indefinite, we call it specific, and we call it nonspecific when it does not. It is widely accepted that three of the four combinations of transparent/opaque with specific/nonspecific are attested in natural language; the names often associated with each combination are specified in Table 1 (cf. von Fintel \& Heim 2011), and examples are discussed below.

The slot for specific-opaque readings is occupied by a question mark in Table 1, as there is controversy regarding the mere existence of this fourth reading (Santorio 2013). While Fodor (1970) claimed the reading is attested, it has since been widely

* Thanks to Itai Bassi, Omri Doron, Patrick Elliott, Danny Fox, Pauline Jacobson, Adèle Mortier, Roger Schwarzschild, Yash Sinha, Margaret Wang, and SALT 31 reviewers and participants for helpful comments and discussion. All errors are my own.

C2021 Benbaji 


\begin{tabular}{|l||cc|}
\hline & Transparent & Opaque \\
\hline \hline Specific $\quad$ (wide-scope) & de re & $?$ \\
\hline Nonspecific (narrow-scope) & the third reading & de dicto \\
\hline
\end{tabular}

Table 1 DPs in intensional contexts

assumed that the reported facts are wrong, and that the three-way distinction between de dicto, de re, and third readings provides us with sufficient empirical coverage of attested readings (Percus 2000, von Fintel \& Heim 2011). More recently, however, Szabó $(2010,2011)$ argued for the existence of a fourth reading, providing a variety of examples judged to be felicitous and true in contexts that make only the specificopaque reading true. Szabó further claims that natural language thus makes available a general mechanism capable of generating fourth reading logical forms (LFs).

Szabó claims that the fourth reading phenomenon is fully general, and that DPs can have the reading relative to both modals and attitudes. In this paper, we focus on one subset of examples, a representative of which is in (1).

a. Context: In Alex's district, judges are elected rather than nominated. Two candidates are trying to win a single vacancy, neither of whom is a judge. The single winner in the upcoming elections will preside (alone) over a hearing in which Alex is a defendant. To make sure that her lawyer prepares for the contingency of either candidate winning, Alex says:

b. There are two judges we could face in this court.

Before we demonstrate how the utterance in (1b) exemplifies the specific-opaque reading, a general observation is in order. The example involves two existential operators: the plural indefinite two and the existential modal could. Given that any two existential quantifiers are scopally commutative - i.e. that $[\exists x \exists y \phi(x, y)]$ and $[\exists y \exists x \phi(x, y)]$ are logically equivalent - we might expect the specific vs. non-specific distinction to be undetectable in this example, as this distinction revolves around the quantificational scope of the operators involved.

The reason the distinction is nevertheless relevant to (1b) is that the utterance makes use of a plural indefinite determiner, and is therefore ambiguous between a reading in which the predicate denoted by the relative clause - that we could face in court - distributes over the plural individual witness of the indefinite and a reading in which it does not, which for convenience we call the non-distributive reading. Distributing a predicate over a plural individual has the effect of universally quantifying over parts of that individual, and this universal quantification is scopally non-commutative with the existential modal in the sentence.

The example in (1b) has four distinct readings, only one of which is true in the 
Restricting the fourth reading

context of (1a). On the de dicto reading, formalized in (2a), there is an accessible possible world in which there are two judges Alex faces in court. This is false given (1a), which makes clear that a single judge will preside over Alex's hearing. On its non-distributive de re construal (2b), the sentence says that two actual judges are such that Alex could face them in court. This is, of course, false in the given context both because of the single judge requirement, and because none of the judicial candidates are judges in actuality. The distributive de re reading requires that there be two actual judges, each of which could preside over Alex's hearing (2c). This reading satisfies the single judge requirement, but nevertheless implies the existence of actual judges and is therefore false in (1a).

The report in (1b) can only be true given (1a) if it expresses the proposition that there are two entities, such that each could be a judge Alex faces in court (2d). This observation can be taken to support the claim that the determiner two in (1b) has to outscope the modal could, while its restrictor is nevertheless interpreted opaquely, in the accessible possible worlds over which the modal quantifies. Note that in (2), and throughout the paper, we represent distributivity as universal quantification over atoms, and use the notation $\exists_{n} x$ to represent $\exists x:|\operatorname{ATOMS}(x)|=n$, i.e. existential quantification over plural individuals consisting of $n$ atoms.

$$
\begin{aligned}
& \text { a. } \lambda w . \exists w^{\prime} \in \operatorname{ACC}(w): \exists_{2} x: \operatorname{judges}_{w^{\prime}}(x) \wedge \text { face-in-court }_{w^{\prime}}(x) \\
& \text { b. } \lambda w . \exists_{2} x: \operatorname{judges}_{w}(x) \wedge \exists w^{\prime} \in \operatorname{ACC}(w): \text { face-in-court }_{w^{\prime}}(x) \\
& \text { c. } \lambda w . \exists_{2} x: \operatorname{judges}_{w}(x) \wedge \forall y \in \operatorname{ATOMS}(x): \exists w^{\prime} \in \operatorname{ACC}(w): \text { face-in-court }_{w^{\prime}}(y) \\
& \text { d. } \lambda w . \exists_{2} x: \forall y \in \operatorname{ATOMS}(x): \exists w^{\prime} \in \operatorname{ACC}(w) \operatorname{judges}_{w^{\prime}}(y) \wedge \text { face-in-court }_{w^{\prime}}(y)
\end{aligned}
$$

The existence of a specific-opaque reading, exemplified by (1), poses a potential problem to what the current orthodoxy tells us about intensionality and scope. Many prominent theories of intensionality (references below) require that a DP take scope below an intensional operator for its restrictor to receive an opaque interpretation. Yet if quantificational scope is determined by LF position, then to take wide quantificational scope a DP must be higher than the intensional operator at LF. Specificity and opacity thus involve conflicting requirements, and unless we reject the assumption that scope is determined structurally, something else must be said about (1). Szabó, for instance, argues that determiners and restrictors can split at LF and have an operator scope between them.

This paper attempts to restrict the scope of the problem by accounting for Szabó's example in (1) and those like it, without departing from standard assumptions about quantification in e.g., Barwise \& Cooper 1981. We argue that a DP can have a specific-opaque reading relative to a modal only when it contains a raising relative clause in which the modal is embedded. As mentioned earlier, the example in (1) represents only a subset of Szabó's data, and the account we present does not cover another subset of apparent fourth readings that does not involve relativization. 
However, we do briefly engage with the rest of Szabó's data, and in particular with examples of the fourth reading relative to attitude predicates that do not contain relative clauses, suggesting that they lend themselves to an alternative explanation if we appeal to previous observations about attitude reports.

The paper is structured as follows: Section 2 presents Szabó's account of the data and our case against it. Section 3 accounts for Szabó's example (1) by invoking independently motivated features of raising relative clauses. Section 4 briefly examines the possibility that examples with attitude verbs are derived from unique features of attitude semantics. Section 5 concludes.

\section{The problem with specific-opaque readings}

The existence of a specific-opaque reading poses a potential problem for at least two prominent theories of intensionality, the modified scope theory (Keshet 2008, Keshet 2010 building on Montague 1973, Ladusaw 1977, Ogihara 1996, a.o.) and the world pronouns framework (Percus 2000, Elbourne 2013, a.o.). In both theories, for a DP to be evaluated in the worlds introduced by an intensional operator -i.e. for it to be opaque - it must reside below the operator at LF. Yet for the DP to be specific, it has to outscope the intensional operator and thus reside above it at LF. If Szabó's examples convince us that our theory of intensionality must be able to generate fourth reading LFs, the conditional dependence of opacity on narrow quantificational scope must be severed. ${ }^{1}$ Yet there does not seem to be an obvious way to do so without denying a basic tenet of Montague semantics, where quantificational scope is determined by position at LF, or of those theories of intensionality, in which DPs are opaque in virtue of their position below an intensional operator at LF.

\subsection{Szabó's solution: bare quantification}

Szabó does not deny the aforementioned tenets, but effectively denies a basic insight of the Generalized Quantifier Theory (GQT, Barwise \& Cooper 1981), according to which determiners denote relations between sets and are represented syntactically as elements that first combine with a restrictor set, and only then with their immediate scope. Szabó claims that the data in (1) constitutes evidence that the expressive power of natural language is greater than GQT would have us believe: Natural language, just like logical ones, allows for bare quantification over the entire domain.

This can be implemented by enriching our syntax with an operation which we term here quantifier splitting (3), that manipulates a structure in which a DP has raised (via QR or otherwise), so that the DP's NP-restrictor is lowered back into its pre-raising position, leaving a bare determiner in the derived position.

1 For a more detailed description of the problem, see Keshet \& Schwarz 2019: 198. 
Restricting the fourth reading

$$
\begin{aligned}
& \text { Quantifier splitting: } \\
& \begin{aligned}
{\left[\mathrm{s}[\mathrm{DP}[D][\mathrm{NP} N]]\left[\lambda_{l}\right]\left[\mathrm{s} \ldots\left[\mathrm{DP} t_{l}\right] \ldots\right]\right] } \\
\qquad\left[\mathrm{s}[\mathrm{DP}[D] \underbrace{[\mathrm{IDENT}]}_{\lambda x . x=x}]\left[\left[\lambda_{l}\right]\left[\mathrm{s} \ldots[\mathrm{DP} \operatorname{THE} \underbrace{\left[\mathrm{IDENT}_{l}\right]}_{\lambda y . y=x_{l}}[\mathrm{NP} N]] \ldots\right]\right]\right]
\end{aligned}
\end{aligned}
$$

The bare determiner D is vacuously restricted by an identity function, while NP is converted into a definite description containing a variable bound by the vacuously restricted D; i.e. NP undergoes trace conversion (Fox 2003, see (16) below). ${ }^{2}$

This system can account for (1) on the assumption that the DP in the example, which seems to receive a specific-opaque interpretation, originates below the intensional operator and raises to a position above it before undergoing quantifier splitting. The truth conditions derived for (1b) from a structure to which quantifier splitting has applied are provided in (4).

$$
\lambda w . \exists_{2} x: \forall y \in \operatorname{ATOMS}(x): \exists w^{\prime}: \text { we-face-in-court } w_{w^{\prime}}\left(\operatorname{lz}\left[\operatorname{judge}_{w^{\prime}}(z) \wedge z=y\right]\right)
$$

Since quantifier splitting always places a definite description in the scope of a vacuously restricted quantifier, and given that definite descriptions are presupposition triggers, this analysis of the fourth reading must be complemented by a theory of presupposition projection in quantificational sentences. The nature of projection out of quantifiers is a notoriously controversial issue, and to avoid it, we adopt the simplifying assumption that presuppositions in the scope of quantifiers can be locally accommodated via the operator in (5a) (cf. Beaver \& Krahmer 2001). This operator, when applied to the scope $\phi$, whose presupposition is $\psi$, of the quantifier in (5b), effectively factors the presupposition into the assertive component as in (5c).

$$
\begin{aligned}
& \text { a. } \llbracket A c c \rrbracket=\lambda p_{s t} \cdot \lambda w \cdot p(w)=1 \\
& \text { b. } \exists_{n} x: \phi_{\psi}(x) \\
& \text { c. } \exists_{n} x: A c c\left(\phi_{\psi}(x)\right)=\exists_{n} x: \psi(x) \wedge \phi(x)
\end{aligned}
$$

\subsection{Against bare quantification}

Szabó's system is not meaningfully restricted: whenever quantifier raising is permitted, so is quantifier splitting. Our argument against bare quantification therefore comes from the observation that the distribution of fourth readings is meaningfully restricted. We show that quantifier splitting generates Szabó's examples at the expense of overgenerating fourth readings when they are clearly unattested. To illustrate this, we construct examples in which (a) the DP can raise above an existential modal, thus permitting quantifier splitting (3); and (b) the DP and the

2 Our implementation of Szabó's account differs slightly from the account in Szabó 2010, 2011 which is partial and does not account for all of Szabó's examples. 
modal are non-commutative, making available a specific and a non-specific reading of the DP. Szabó wrongly predicts such examples to have specific-opaque readings.

Plural indefinites in subject position can outscope and distribute over operators they command, as is evident from example (6), which can be true when a department has two lecturers capable of teaching phonology, even if co-teaching is not permitted.

(6) Two professors could teach Introduction to Phonology.

Given that distributivity is available, the indefinite subject is scopally non-commutative with the existential modal. Furthermore, on the standard assumption that the subject originates below the modal and raises to its surface position, quantifier splitting should be permitted in these cases. Szabó's bare quantification account thus predicts that subject-position existential quantifiers will receive a specific-opaque reading relative to existential modals.

We test this prediction by slightly modifying Szabó's original context and example (1), as in (7).

(7) a. Context: Alex is awaiting the judicial elections as in (1a), only this time there are five candidates. Alex believes only two of them stand a chance, and instructs her lawyer to prepare for either of the two winning.

b. Two candidates could end up on the bench. (The others have no chance.)

c. \# Two judges could end up on the bench.

d. There are two judges who could end up on the bench.

Nothing prevents the subject position quantifier in (7c) from distributing over the modal - this is exactly what happens in (7b) - and nothing in principle blocks quantifier splitting (3). Yet (7c) is nevertheless infelicitous given (7a), unlike the felicitous example in (7d), which maintains the structure of Szabó's original example (1b). Therefore, bare quantification overgenerates a specific-opaque reading for (7c) and fails to account for the disparity between (7c) and (7d).

Before we proceed, note that the minimal modification in (8b) of Szabó's example, repeated in (8a), cannot by itself be used to argue against bare quantification.

a. There are two judges we could face in this court.

b. \# We could face two judges in this court.

Granted, only (8a) is felicitous in the judicial elections context (1a), which makes only the fourth reading true, while (8b) is felicitous only if two judges will preside over Alex's case. However, since the modal and the indefinite are scopally commutative, the de dicto reading of (8b) is equivalent to its fourth reading when the object position indefinite fails to inverse scope and distribute. And it has been illustrated independently that inverse-scope object-distributive readings of indefinites are disallowed or dispreferred (Steedman 2011, Križ \& Maldonado 2018 and references therein). Szabó can thus attribute the disparity in (8) to the absence of distributivity. 
Restricting the fourth reading

Another quantifier that is scopally non-commutative with existential modals, and which can QR to a position above operators that command it, is the universal quantifier every. Example (9), for instance, can be true even if Sue has no time to read all papers on the syllabus, as long as she is capable of reading each of them.

(9) Sue could read every linguistics paper on the syllabus.

It seems then that Szabó predicts that a universal quantifier will have the fourth reading relative to an existential modal that commands it. Testing this prediction, however, requires further assumptions about the projection of presuppositions from the scope of universal quantifiers, and the theoretical choices we make will affect the prediction itself. In what follows, we test the prediction assuming local accommodation via the operator in (5a).

Given our assumption of local accommodation, when an accommodation operator is applied to the quantifier's scope in $\forall x: \phi_{\psi}(x)$ (where $\psi$ is a presupposition of the scope $\phi$ ), the statement will assert that $\forall x: \psi(x) \wedge \phi(x)$. Since quantifier splitting creates quantifiers with vacuous restrictors, when the operation is applied to universals it will produce structures that give rise to truth conditions that are quite strong, as they require the scope of the quantifier to be true for every individual in the domain. Therefore, one could plausibly claim that while our grammar can generate specific-opaque LFs for universal statements, this reading is rarely attested (if ever), as its truth conditions are too strong for it to ever be true. For instance, a bare quantification construal of the variation on Szabó's example in (10) will give rise to the impossibly strong truth conditions according to which for every individual in the domain, there is a world in which it is a judge and we face that judge in court.

(10) We could face every judge in this court.

However, if quantifiers that have undergone quantifier splitting and are therefore vacuously restricted by an identity function can nevertheless be contextually restricted, Szabó predicts universal statements to have felicitous and true specificopaque readings. This is because contextual restriction will allow us to restrict the domain of quantification to individuals that make the assertion and accommodated presupposition true. We can test this prediction by construing a context that sufficiently restricts the domain of quantification, as in (11).

(11) a. Context: Alex is awaiting the judicial elections. The list of candidates has not yet been publicized. Her lawyer brings her an early copy, saying:

b. \# We could face every judge in this court.

c. \# Every judge could end up on the bench.

d. Everyone is a liberal. 
Neither of (11b-c) can mean that every domain entity on the list obtained by the lawyer could be a judge that Alex faces in court. This is despite the fact that the context in (11a) can surely restrict quantification to those on the list, as is indicated by the felicity of (11d) in that context, when used to indicate that every listed candidate is a liberal. The prediction made by the bare quantification account with respect to universal statements, together with our assumptions about accommodation and contextual restriction, is therefore not borne out.

Further discussion of our assumptions regarding presupposition accommodation will take us too far from the focus of this paper. As for the assumption that bare quantification can be contextually restricted, however, it is important to note that Szabó himself makes this assumption to account for a data point that we have not discussed (see Szabó 2011: 274 for further discussion). Finally, the argument against bare quantification from subject position indefinite DPs in (7) stands without any potentially controversial assumptions.

\section{A restrictive account of specific-opaque readings}

To illustrate the specific-opaque reading, Szabó introduced the judicial elections context, where the candidates are not actual judges (opacity), and only one will eventually be elected (specificity+distributivity). In the previous section, we show that while Szabó's example (8a) is felicitous in this context, a slight manipulation renders it infelicitous $(7 \mathrm{c}, 8 \mathrm{~b}, 11 \mathrm{~b}-\mathrm{c})$. We claim next that the relevant structural difference between Szabó's felicitous example and the infelicitous ones above is that only the former contains a relative clause (RC) embedding an intensional operator. We further argue that specific-opaque readings are restricted to relativization environments, and are achieved by invoking independently motivated assumptions about raising RCs.

\subsection{Some structural properties of raising $\mathrm{RCs}$}

A prominent analysis of RCs in contemporary syntax argues that they are ambiguous between a raising structure, in which the nominal element modified by the RC originates RC-internally and raises to its surface position, and an external-head structure, in which that nominal element originates RC-externally (cf. Carlson 1977, Bhatt 2002, Heim 1987, Sauerland 1998). We will argue that a specific-opaque reading of a DP relative to a modal operator is only available when the modal is in a raising $\mathrm{RC}$ headed by the $\mathrm{DP}$, relying on two properties of raising $\mathrm{RCs}$ when making the argument: (1) the ability of the determiner in a RC-embedding DP to originate RC-externally; and (2) the ability of the head NP in a raising RC to be interpreted inside the RC, as if it had not raised; i.e. its ability to undergo reconstruction. 
Restricting the fourth reading

\subsubsection{NPs raise, not DPs}

There are convincing arguments that the determiner head of a RC-embedding DP does not originate RC-internally (Partee 1975, Heim \& Kratzer 1998); i.e. that (13a) is preferable to (13b) as the syntactic structure of the DP in (12).

(12) The book that Elena wrote
a. $\left[{ }_{\mathrm{DP}}\left[{ }_{\mathrm{D}}\right.\right.$ the $]\left[\left[_{\mathrm{NP}}\right.\right.$ book $]\left[_{\mathrm{RC}}\right.$ that Elena wrote $\left[_{\mathrm{NP}}\right.$ book $\left.\left.\left.]\right]\right]\right]$
b. $\left[\mathrm{DP}\left[\mathrm{DP}\right.\right.$ the book] ${ }_{\mathrm{RC}}$ that Elena wrote $[\mathrm{DP}$ the book] $\left.]\right]$

One such reason is that (13b) leads to type mismatches, on the assumption that RCs denote $\langle e, t\rangle$-type functions. Whether the raised DP in (13b) is of type $\langle e t, t\rangle$ or of type $e$ (cf. Heim \& Kratzer 1998), functional application at the highest node in the structure will yield a truth-value, when in fact DPs with embedded RCs inherit the type resulting from merging their determiner $\mathrm{D}$ with an $\langle e, t\rangle$-type function.

Further support for (13a) comes from the presuppositions of RC-embedding DPs. The structure in (13b) wrongly predicts undefinedness when the function denoted by the NP is not in the domain of the determiner. The one in (13a) predicts undefinedness only when the function obtained by intersecting the denotations of the NP and the RC is not in the domain of the determiner (Partee 1975: 230-31). According to (13b), then, (12) is predicted to be undefined when more than one book is contextually salient. But this is not so. All that is required for the phrase to be defined is that there be only one contextually salient book that Elena wrote, as predicted by (13a). We are therefore justified in assigning (12) the structure in (13a).

\subsubsection{Raised NPs can reconstruct}

The second feature of raising RCs that we rely on in our analysis is the ability of the $\mathrm{NP}$ heading a $\mathrm{RC}$ to reconstruct into its $\mathrm{RC}$-internal merging position. The literature on reconstruction into RCs is too vast to be reviewed here, and we refer the reader to a summary of arguments in support of reconstruction in Bhatt (2002: 46-56). In our account below, we use the semantics for reconstructed RCs proposed by Bassi \& Rasin (2018), in the spirit of Heim's (2018) analysis of functional readings of questions. $^{3}$ The starting point for Bassi \& Rasin is an ambiguity detected by Grosu \& Krifka (2007) in examples like (14).

(14) The gifted mathematician that Bill claims he is should be able to solve this problem in no time.

3 Bassi \& Rasin argue that reconstruction is a syntactic phenomenon, rather than a semantic one. Discussing the semantic $v s$ syntactic reconstruction debate is beyond the scope of this paper, and so we leave the question of whether and how our account of the fourth reading can be recast in terms of semantic reconstruction for future research. 
According to one reading of (14), Bill claims that he is a gifted mathematician, and given that claim, he should be able to solve the problem. On this reading, the phrase gifted mathematician is evaluated opaquely relative to the attitude predicate claim, and the sentence does not imply the existence of a gifted mathematician in actuality. On the other less salient reading, there is a certain actual gifted mathematician, say Hilbert, who should be able to solve the problem, and Bill claims that he is Hilbert.

The opaque reading of gifted mathematician presents us with an apparent syntaxsemantics mismatch. Since this reading only implies the existence of a gifted mathematician in Bill's claim-worlds, the NP gifted mathematician must be interpreted in the scope of the attitude verb. However, its surface position outscopes the attitude verb. Therefore, the opaque reading of (14) cannot be derived by applying standard interpretation rules to the surface syntax of the sentence.

Bassi \& Rasin account for this mismatch by assuming that the RC in (14) has the raising structure discussed in (13a); i.e. one in which the phrase gifted mathematician originated clause internally, and raised to its surface position. They furthermore argue that to receive an opaque interpretation, that phrase reconstructs into its RC-internal position. Assuming the copy theory of movement (Chomsky 1995), the process Bassi \& Rasin propose consists of three steps. First, the nominal is internally-merged in a RC-external position, leaving a $\lambda$-binder along the way (15). Second, the RC-internal copy of the nominal undergoes trace conversion (16) (Fox 2003); an operation consisting of insertion of a bound variable to the noun phrase (16a), and replacement of the nominal's determiner with the definite article (16b). ${ }^{4}$ Finally, the higher, pronounced copy of the nominal at the top of the movement chain is deleted, and the RC obtained in (17) can be merged with a determiner.

Internal-merge of NP ( "raising”):

$[\mathrm{RC} \cdots[\mathrm{DP}[\mathrm{D}][\mathrm{NP} \mathrm{N}]]] \rightarrow\left[\left[\mathrm{NP}_{\uparrow} \mathrm{N}\right]\left[\left[\lambda_{i}\right]\left[\mathrm{RC} \cdots\left[\mathrm{DP}[\mathrm{D}]\left[\mathrm{NP}_{\mathrm{NP}} \mathrm{N}\right]\right]\right]\right]\right]$

(16) Trace conversion:

a. $\left[\left[\lambda_{i}\right]\left[{ }_{\mathrm{RC}} \cdots\left[{ }_{\mathrm{DP}}[\mathrm{D}]\left[{ }_{\mathrm{NP}} \mathrm{N}\right]\right]\right]\right] \rightarrow\left[\left[\lambda_{i}\right]\left[{ }_{\mathrm{RC}} \cdots\left[{ }_{\mathrm{DP}}[\mathrm{D}]\left[{ }_{\mathrm{NP}}[\mathrm{N}]\left[\lambda y \cdot y=x_{i}\right]\right]\right]\right]\right]$

b. $\quad \rightarrow\left[\left[\lambda_{i}\right]\left[{ }_{\mathrm{RC}} \cdots\left[\mathrm{DP}[\mathrm{THE}]\left[\mathrm{NP}[\mathrm{N}]\left[\lambda y \cdot y=x_{i}\right]\right]\right]\right]\right]$

(17) Higher copy deletion:

$$
\begin{aligned}
{\left[\left[{ }_{\mathrm{NP}} \mathrm{N}\right]\left[\left[\lambda_{i}\right]\left[{ }_{\mathrm{RC}} \cdots\left[\mathrm{DP}_{\mathrm{DP}}[\mathrm{THE}]\left[\mathrm{NP}[\mathrm{N}]\left[\lambda y \cdot y=x_{i}\right]\right]\right]\right]\right]\right] } & \\
& \rightarrow\left[\left[\lambda_{i}\right]\left[\mathrm{RC}_{\mathrm{RC}} \cdots\left[\mathrm{DP}[\mathrm{THE}]\left[\mathrm{NP}[\mathrm{N}]\left[\lambda y \cdot y=x_{i}\right]\right]\right]\right]\right]
\end{aligned}
$$

\subsection{Specific-opaque LFs}

We reviewed the motivation for the following assumptions: First, NPs raise in raising RCs, while their determiners originate RC-externally; and second, raising NPs can

4 Given that this determiner is always replaced, we do not know what it was in the first place. 
reconstruct into their RC-internal position, where they undergo trace conversion. Szabó's example, repeated in (18), consists of a plural indefinite DP and an intensional raising $\mathrm{RC}$ that distributes over the plural individual witness of the indefinite. ${ }^{5}$

(18) There are two judges we could face in this court.

Given our first assumption, the distributive element associated with the indefinite will take wide scope relative to the intensional operator, as it originates outside the $\mathrm{RC}$, higher than the operator. Given our second assumption, the NP that appears in a $\mathrm{RC}$-external position can in fact reconstruct into its $\mathrm{RC}$-internal one.

When reconstruction occurs, the determiner outscopes the intensional operator, while the NP is interpreted in the worlds introduced by the operator. This scopal configuration is exactly what we need to derive the fourth reading. A reconstruction LF is provided in (19), with the truth conditions derived via this LF in (20). For illustrative purposes we make use of a distributive operator with the semantics in (21), but the analysis does not hinge on this particular implementation of distributivity.

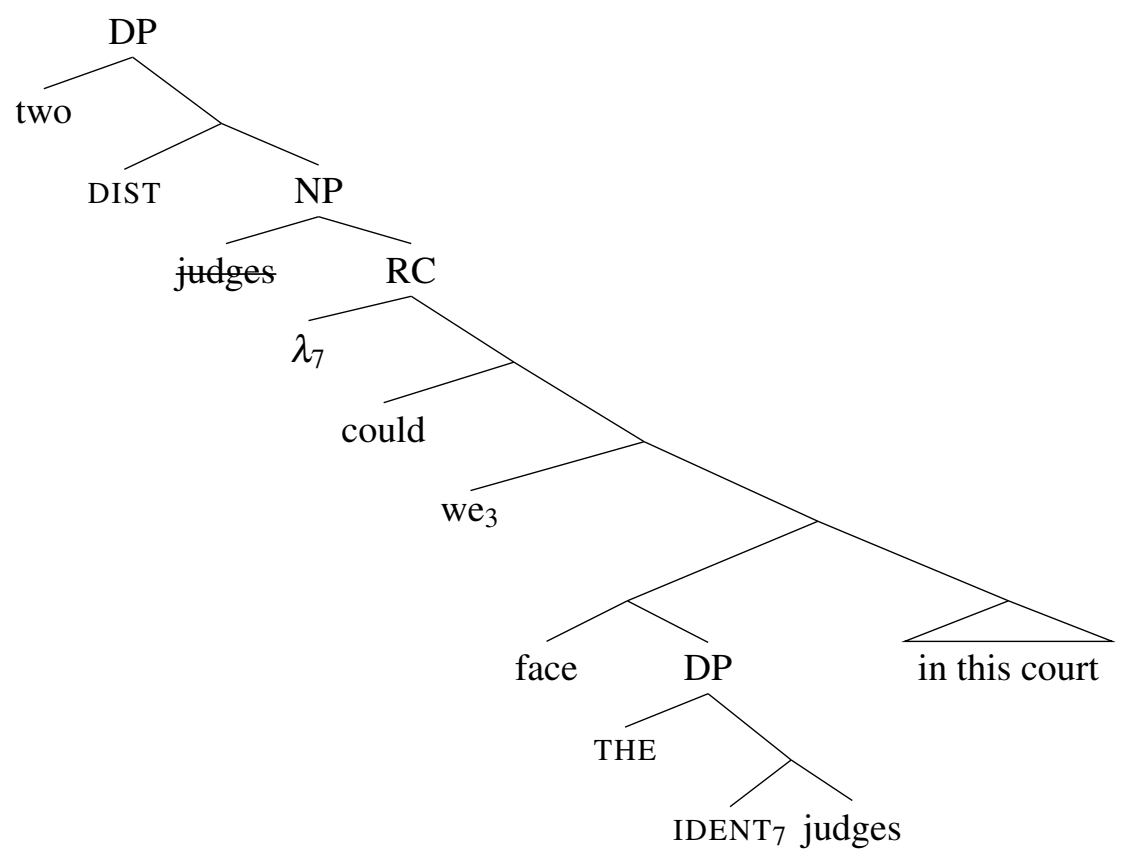

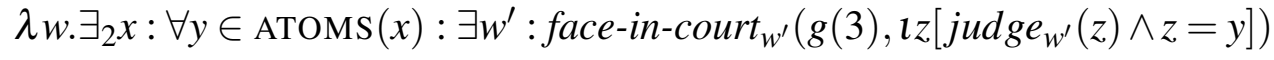

$$
\begin{aligned}
& \llbracket \mathrm{DIST} \rrbracket=\lambda f_{\langle e, t\rangle} \cdot \lambda x_{e} . \forall y \in \operatorname{ATOMS}(x): f(y)
\end{aligned}
$$

The analysis illustrates that the fourth reading of modal claims can be accounted for without Szabó's bare quantification mechanism. It is superior to that account as

5 Note that for simplicity of exposition we treat the numeral itself as a determiner of type $\langle e t,\langle e t, t\rangle\rangle$, but the analysis can be innocuously reformulated if the numeral is a $\langle e, t\rangle$-type modifier under an existential determiner (cf. Bylinina \& Nouwen 2020). 
it avoids overgeneration of unattested readings. Of the examples we have examined so far, those that lack a fourth reading $(7 \mathrm{c}, 8 \mathrm{~b}, 11 \mathrm{~b}-\mathrm{c})$ also lack a $\mathrm{RC}$ altogether. Therefore, if the semblance of bare quantification - and thus of a fourth reading can only be achieved when DPs dominate a raising RC containing an intensional operator, the unavailability of the fourth reading in these examples is predicted.

Furthermore, the analysis correctly predicts that in the presence of an intensional raising $\mathrm{RC}$, a fourth reading will be available for any determiner that is non-commutative with the RC-internal intensional operator. To see this, consider the examples and context in (22).

(22) a. Context: Alex's lawyer obtains a list of potential judicial election candidates and presents it to Alex.

b. Every judge we could face in court is on this list.

c. No judge we could face in court is on this list.

$\mathrm{d}$. The judge we will face in court is on this list.

Alex's lawyer can use (22b) to say that the list is an exhaustive list of candidates. Alex can reject that claim with (22c), and the lawyer can reply with (22d). Crucially, all three utterances do not discuss actual judges, and only the fourth reading is true.

Our analysis does not posit any mechanism not already assumed by Bassi \& Rasin (2018). In fact, the only novel contribution in this section is the observation that Grosu \& Krifka's RC in (14), analyzed by Bassi \& Rasin, is just a special case of a larger class of RCs; namely, fourth reading RCs. Given that determiners dominating raising $\mathrm{RCs}$ do not originate $\mathrm{RC}$-internally (and therefore cannot reconstruct), we predict that RCs will never have opaque readings in which the determiner scopes below a RC-internal intensional operator. Determiners dominating RCs are always specific relative to $\mathrm{RC}$-internal intensional operators.

While Szabó's example (18) has two distinct paraphrases, one ostensibly corresponding to a non-specific opaque reading, and one to the specific-opaque reading, this stems from the fact that the determiner and operator in the example (two and could) are scopally commutative. Therefore, on the non-distributive construal, the specific-opaque reading and the non-specific one are equivalent. What we took to be the non-specific reading of (18), then, is merely its non-distributive fourth reading.

\subsection{Predictions}

If fourth readings are only derived from raising $\mathrm{RCs}$, they are predicted to be unavailable for DPs with RCs for which a raising construal is independently blocked. We illustrate next that this prediction is borne out. 
Restricting the fourth reading

\subsubsection{Resumptive pronouns in Modern Hebrew}

Doron (1982) discovered an interpretative difference between Modern Hebrew RCs with a gap in their object position (23a) and those with a resumptive pronoun (23b).

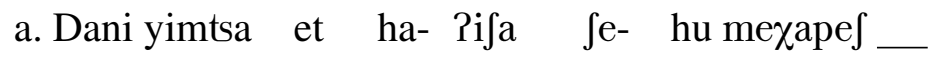
Dani will.find ACC the- woman that- he seeks

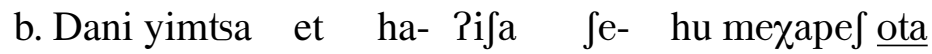
Dani will.find ACC the- woman that- he seeks her.RP

'Danny will find the woman that he seeks.'

Example (23a) is ambiguous between a reading according to which there is a specific woman Danny is looking for and is expected to find, and a reading according to which Danny has no specific woman in mind. Rather, he has some conception of a woman and is expected to find a woman who fits that conception. The presence of a resumptive pronoun in (23b), Doron noticed, blocks the latter reading.

Sichel (2014) uses this example (among others) to argue that the presence of a resumptive pronoun blocks reconstruction into a $\mathrm{RC}$. If this is the case, the data in (23) comes for free as long as we assume that the difference between the readings reflects relative scope; i.e. that the ambiguity in (23a) is the result of two LFs, one in which the relative head reconstructs below the RC-internal intensional verb, and another in which it outscopes that verb. If reconstruction is blocked by resumption, then so are readings that require wide scope for the intensional verb.

Together with Sichel's proposal, our account of the fourth reading predicts the reading to be unavailable for DPs with RCs whose gap position is occupied by a resumptive pronoun. This is indeed the case. The Hebrew version of (18) in (24) is infelicitous in the judicial elections context (1a) when it contains a resumptive pronoun, in which case it can only be interpreted de re. When the resumptive pronoun is omitted, (24) behaves just like its English counterpart.

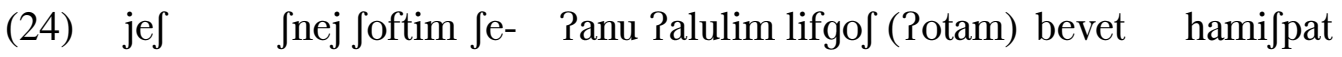
there.are two judges that-we could meet them.RP in.house the.law

'There are two judges we could face in court.'

\subsubsection{Extraposition}

Hulsey \& Sauerland (2006) argue that extraposition of a RC past a right-adjoined adverb blocks a raising construal of the RC. They show that extraposition renders ungrammatical RCs that require their heads to reconstruct for independent reasons; including RCs with head NPs that form idioms with RC-internal material (25a), and RCs whose heads contain anaphors that must be bound by RC-internal elements to satisfy Condition A (25b). Both configurations block extraposition (26a-b). 
(25) a. Det $\left[{ }_{N P} \text { idiom part } \ldots\right]_{j}\left[R C \ldots\right.$ idiomatic environment $\left.t_{j} \ldots\right]$ Idiom chunks

b. Det $\left[N P \ldots \text { anaphor }_{i} \ldots\right]_{j}\left[R C \ldots\right.$ R-expression $\left.{ }_{i} \ldots \mathrm{t}_{j}\right] \quad$ Condition A

(26) a. Mary praised the headway (*last year) that John made.

b. Mary saw the picture of himself (* $^{*}$ yesterday) that Bob $_{i}$ likes.

If extraposition blocks raising structures, the fourth reading should be unavailable when the RC containing the intensional operator has been extraposed.

The prediction is indeed borne out. This is illustrated with the variation on the judicial elections context in (27). It seems that in the context of (27a), Alex cannot felicitously utter (27b) in conversation with her lawyer, as predicted if extraposition in (27b) blocks raising, which is in turn is a prerequisite for the fourth reading. The alternative without extraposition in (27c) seems felicitous in the provided context.

(27) a. Context: Alex is awaiting the results of a judicial elections to see who will preside over her court case. She is a well-known politician and is encouraged by her lawyer to use her political platform to praise all judicial candidates, so that whoever wins will judge her favorably.

b. \# I praised every judge yesterday that we could face in court.

c. I praised every judge that we could face in court.

\subsubsection{Condition C}

The acceptability of RCs whose heads contain R-expressions sharing indices with an RC-internal pronoun, e.g., (28), is evidence that RCs cannot have only raising structures. If we construe these RCs as involving raising (29), their heads could potentially reconstruct into a position below a co-referential pronoun, in violation of Condition C. If reconstruction feeds Condition C (as argued in Romero 1998 and Fox 1999), RCs of this sort are expected to be unacceptable - contrary to fact.

The picture of $\mathrm{John}_{i}$ that he $\mathrm{i}_{i}$ likes

Therefore, such RCs cannot have raising structures, and we predict that they will not give rise to fourth readings either. It seems that this prediction is indeed borne out. To see this, consider yet another variation on Alex's story.

(30) a. Context: Alex is a lawyer accused of wrongdoing and awaiting trial. Two candidates in the judicial elections that will determine who will preside over the trial are currently colleagues of hers at her law firm.

b. ?\# There are two judges from Alex's law firm that she could face in court.

c. There are two judges from her law firm that Alex could face in court. 
Restricting the fourth reading

We predict (30b) to be infelicitous in the context of (30a), in which only the fourth reading is true, as the fourth reading requires a raising structure that is blocked by Condition C. We expect (30c) to be felicitous, on the other hand, since its RC head contains no R-expression, but merely a pronoun, whose binding inside the $\mathrm{RC}$ does not violate binding conditions. While judgments are delicate, there does seem to be a disparity between the utterances, illustrating that the prediction is borne out.

\section{A note on specific-opaque readings without relative clauses}

We analyzed one data point - repeated in (31) - that Szabó $(2010,2011)$ presents to argue for the existence of a fourth, specific-opaque reading, and showed that an analysis that relies on the presence of a $\mathrm{RC}$ in the example is superior to one that assumes a generally available quantifier splitting operation. This example is the only data point that Szabó presents to exemplify the fourth reading relative to modals. However, as mentioned in Section 1, Szabó provides further data involving attitude predicates to illustrate the existence of the fourth reading, and these are not amenable to our analysis above.

The example in (32) is Szabó's example of the fourth reading relative to the attitude predicate believe.

(31) There are two judges we could face in this court.

(32) The fourth reading relative to a propositional attitude:

a. Context: While under questioning by the police, Alex is presented with a binder of photos of people who she takes to be her neighbors, and is asked to identify thieves. She points to several photos, but does not keep track of how many. The police officer does keep track, and later reports:

b. Alex believes that eleven thieves live in her building.

The utterance in (32b) seems true in the context of (32a). However, since Alex did not count the allegations she made, she does not have a de dicto belief that eleven is the number of thieves in her building, and since the photos she pointed to might not depict actual thieves, the de re and third readings are both false, as they involve transparent interpretation of a DP's restrictor. The only true reading of the utterance given the context is one according to which eleven things are such that Alex believes them to be thieves living in her building. In other words, the determiner eleven needs to be interpreted outside the scope of the attitude believe, while its restrictor thieves must nevertheless receive an opaque interpretation, in Alex's belief-worlds.

Our analysis is silent about (32). In fact, if we claim that fourth readings are restricted to relativization environments, we undergenerate this example and others containing attitude predicates and lacking a RC. However, if we are correct that 
(31) should not be generated via bare quantification, and examples like (31) are the only cases exemplifying the fourth reading relative to modal operators, then the phenomenon underpinning Szabó's argument for bare quantification is no longer fully general. Rather, it seems restricted to attitude predicates. Perhaps, then, the right approach to (32), is one that appeals to the unique features of attitudes to derive specific-opaque readings of attitude reports. Pursuing an account of this sort for (32) is beyond the scope of this paper. However, we end with highlighting the merits of such an approach.

First note that, as Keshet \& Schwarz (2019) point out, if in the police-questioning context (32a) Alex believes of eleven entities that they are thieves living in her building, then upon reflection on her individual beliefs she would have to agree with the de dicto reading of (32b) if she is at all rational. Perhaps, then, (32b) is true in the context above on its de dicto reading after all, as Alex's different beliefs considered together entail this reading. Szabó's objection to a reduction of the fourth reading to the de dicto one in the case of (32b) notes that beliefs are generally not deductively closed (for instance, we do not believe every valid formula), despite us sometimes treating them as such. "If we don't want to take the blame when the simplifying assumption of logical omniscience leads to unacceptable predictions we should not take credit when it accidentally delivers the right result" (Szabó 2010: 23).

However, we need not wait for a comprehensive solution to the deduction problem, one which explains why belief seems to be closed under deduction in some cases but not in others, to derive specific-opaque readings from the properties of belief. This is because there is another, more restricted data set involving attitude predicates that seems suspiciously similar to Szabó's data, and a successful analysis of that data set, might suffice to explain away (32) without bare quantification.

The data set exemplifies a phenomenon termed revisionist reporting by Blumberg \& Lederman (2021), in which a report that an agent believes a proposition $p$ is taken as true even if $p$ is not entailed by the agent's beliefs, as long as it is entailed by a revision of these beliefs in light of the reporter's presuppositions. Consider (33):

a. Context: Peter believes any six-year-old can learn how to play tennis in ten lessons. He does not know Alona, a six-year-old, whose parents are aware of Peter's views on tennis pedagogy. Alona's mother utters:

b. Peter believes Alona can learn how to play tennis in ten lessons.

Blumberg \& Lederman propose that (33b) is felicitous in (33a), as (loosely speaking) the information state obtained from revising Peter's background information with that of Alona's mother, entails the prejacent of the attitude in (33b).

Now reconsider Szabó's police-questioning context. There, we assumed Alex lacks the de dicto belief that there are eleven thieves in her building, as she did not count her allegations. The reporter in that context (the police officer) counted eleven 
Restricting the fourth reading

of them. Therefore, the officer's information state makes true the proposition that Alex pointed to eleven photos during questioning, while in Alex's information state every person she pointed to during questioning (however many that is) is a thief. A mechanism that revises the information state relative to which the truth of an attitude report is evaluated to account for Blumberg \& Lederman's example, can be extended to account for the truth of (32b) in Szabó's context. This is because the police officer's belief that eleven allegations were made, when complementing Alex's information state, entails the prejacent proposition in (32b), according to which there are eleven thieves in Alex's building. ${ }^{6}$

In response to Szabó, Ben-Yami (2014) pointed out that slightly modifying the police-questioning context as in (34), renders example (32b) infelicitous.

(34) a. Context: Alex is under questioning again. This time she counts her allegations, but mistakenly takes three photos of alleged thieves to depict the same person. Upon leaving the police station, then, Alex has formed the (de dicto) belief that there are nine thieves in her building.

b. Alex believes that nine thieves live in her building.

c. \# Alex believes that eleven thieves live in her building.

The utterance in (34b) seems to be felicitous in Ben-Yami's context, while Szabó's original utterance (32b), repeated in (34c), seems degraded (Ben-Yami 2014: 180). Under Szabó's account, it is unclear why we have this intuition. Everything that made (34c) true in Szabó's context (32a) is still there in Ben-Yami's (34a). Granted, while the de dicto reading of (34c) is false in both contexts, it is false for different reasons: in Szabó's context, Alex has no belief regarding the number of alleged thieves, while in Ben-Yami's, she has a false one. But if (34c) has a LF representation that renders it felicitous and true in Szabó's context, why should this matter?

If Szabó's example is a case of revisionist reporting, the puzzle can be resolved by constraining the mechanism that derives these reports, so that it will not allow revision of a certain information state with a proposition that is incompatible with it. This constraint distinguishes Szabó's context, where the officer's belief is compatible with Alex's, from Ben-Yami's modification where they contradict each other. BenYami's point thus further motivates a revisionist reporting account of (32b).

\section{Conclusion}

The existence of a fourth, specific-opaque reading has been claimed to pose a problem for current theories of intensionality. This paper attempts to restrict the

6 Of course, implementing this revision is a complicated task, and we point the reader to the discussion in Blumberg \& Lederman (2021), as well as in Percus (2020: 24), where "replacement" operators are used to manipulate the prejacents of attitude predicates to account for revisionist reporting. 
scope of the problem by taking a closer look at the distribution of these readings, suggesting that the phenomenon is not fully general. More specifically, we show that the fourth reading relative to modals is restricted to relativization environments.

Our findings strongly suggest that natural language does not make generally available a mechanism that generates these readings, as it does for the other three commonly accepted readings. Rather, independently motivated features of relativization environments conspire to create the effect of specific-opaque DPs in modal environments. We briefly suggest that other independently motivated assumptions regarding attitude predicates might similarly conspire to create that effect in attitude reports, though we do not implement this proposal.

Our analysis is in opposition to that in Szabó 2010, 2011, which derives specificopaque readings by positing a syntactic operation that splits quantifiers from their restrictors at LF. We not only argue that quantifier splitting is not required to account for the data, but also that it is not allowed, as it overgenerates unattested readings. It has been argued, however, that natural language requires quantifier splitting elsewhere. For instance, Rullmann \& Beck (1998) claim that which-phrases in de dicto readings of which-questions contribute bare existential quantifiers in their derived positions, while their restrictors undergo trace conversion in their original merging positions. If this is correct, one should wonder what in the which-question environment renders quantifier splitting available, and how this mechanism can be constrained so that it does not overgenerate the examples we provide in this study. We leave these more general questions for future investigation.

\section{References}

Barwise, Jon \& Robin Cooper. 1981. Generalized quantifiers and natural language. Linguistics and Philosophy 4. 159-219. doi:10.1007/BF00350139.

Bassi, Itai \& Ezer Rasin. 2018. Equational-intensional relative clauses with syntactic reconstruction. In Sinn und Bedeutung (SuB), vol. 22, 143-159. doi:10.21248/zaspil.60.2018.459.

Beaver, David. 1994. When variables don't vary enough. Semantics and Linguistic Theory (SALT) 4. 35-60.

Beaver, David \& Emiel Krahmer. 2001. A partial account of presupposition projection. Journal of Logic, Language and Information 10(2). 147-182.

Ben-Yami, Hanoch. 2014. Bare quantifiers? Pacific Philosophical Quarterly 95(2). 175-188. doi:10.1111/papq.12023.

Bhatt, Rajesh. 2002. The raising analysis of relative clauses: Evidence from adjectival modification. Natural Language Semantics 10. 43-90. doi:10.1023/A:1015536226396. 
Restricting the fourth reading

Blumberg, Kyle \& Harvey Lederman. 2021. Revisionist reporting. Philosophical Studies 178. 755-783. doi:10.1007/s11098-020-01457-4.

Bylinina, Lisa \& Rick Nouwen. 2020. Numeral semantics. Language and Linguistics Compass 14(8). 1-18. doi:10.1111/lnc3.12390.

Carlson, Greg N. 1977. Amount relatives. Language 53. 520-542. doi: $10.2307 / 413175$.

Chomsky, Noam. 1995. The Minimalist Program. Cambridge: MIT press.

Doron, Edit. 1982. On the syntax and semantics of resumptive pronouns. In Texas Linguistic Forum, vol. 19, 1-48. University of Texas.

Elbourne, Paul. 2013. Definite Descriptions. Oxford: Oxford University Press.

von Fintel, Kai \& Irene Heim. 2011. Intensional Semantics. MIT lecture notes.

Fodor, Janet. 1970. The linguistic description of opaque contexts: MIT PhD dissertation.

Fox, Danny. 1999. Reconstruction, binding theory, and the interpretation of chains. Linguistic Inquiry 30. 157-196. doi:10.1162/002438999554020.

Fox, Danny. 2003. On logical form. In Randall Hendrick (ed.), Minimalist Syntax, 82-123. Malden, MA: Blackwell. doi:10.1002/9780470758342.ch2.

Grosu, Alexander \& Manfred Krifka. 2007. The gifted mathematician that you claim to be: Equational intensional 'reconstruction' relatives. Linguistics and Philosophy 30. 445-485. doi:10.1007/s10988-008-9022-5.

Heim, Irene. 1987. Where does the definiteness restriction apply: Evidence from the definiteness of variables. In Eric J. Reuland \& Alice G. B. ter Meulen (eds.), The Representation of (In)definiteness, 21-42. Cambridge, MA: MIT Press.

Heim, Irene. 2018. Functional readings without type-shifted noun phrases. In Manfred Krifka \& Mathias Schenner (eds.), Reconstruction Effects in Relative Clauses, 283-302. Berlin: De Gruyter.

Heim, Irene \& Angelika Kratzer. 1998. Semantics in Generative Grammar. Oxford: Blackwell.

Hintikka, Jaakko. 1962. Knowledge and Belief: An Introduction to the Logic of the Two Notions. Ithaca, NY: Cornell University Press.

Hulsey, Sarah \& Uli Sauerland. 2006. Sorting out relative clauses. Natural Language Semantics 14. 111-137. doi:10.1007/s11050-005-3799-3.

Keshet, Ezra. 2008. Good intensions: Paving two roads to a theory of the de relde dicto distinction: MIT PhD dissertation.

Keshet, Ezra. 2010. Split intensionality: A new scope theory of de re and de dicto. Linguistics and Philosophy 33. 251-283. doi:10.1007/s10988-011-9081-x.

Keshet, Ezra \& Florian Schwarz. 2019. De re/de dicto. In Jeanette Gundel \& Barbara Abbott (eds.), The Oxford Handbook of Reference, 167-202. Oxford: Oxford University Press.

Križ, Manuel \& Mora Maldonado. 2018. An experimental note on distributivity and 
scope. Manuscript, LSCP.

Ladusaw, William. 1977. Some problems with tense in PTQ. In Texas Linguistic Forum, vol. 6 1977, 90-102. University of Texas.

Montague, Richard. 1973. The proper treatment of quantification in ordinary English. In Jaakko Hintikka, Julius Moravcsik \& Patrick Suppes (eds.), Approaches to Natural Language, 221-242. Dordrecht: Reidel.

Ogihara, Toshiyuki. 1996. Tense, Attitudes, and Scope. Springer.

Partee, Barbara. 1975. Montague grammar and transformational grammar. Linguistic Inquiry 6(2). 203-300.

Percus, Orin. 2000. Constraints on some other variables in syntax. Natural Language Semantics 8. 173-229. doi:10.1023/A:1011298526791.

Percus, Orin. 2020. Index-dependence and embedding. The Wiley Blackwell Companion to Semantics. 1-31. doi:10.1002/9781118788516.sem096.

Romero, Maribel. 1998. Focus and reconstruction effects in wh-phrases: University of Massachusetts Amherst PhD dissertation.

Rullmann, Hotze \& Sigrid Beck. 1998. Presupposition projection and the interpretation of 'which'-questions. Semantics and Linguistic Theory (SALT) 8. 215-232. doi:10.3765/salt.v8i0.2811.

Santorio, Paolo. 2013. Descriptions as variables. Philosophical Studies 164. 41-59. doi:10.1007/s11098-013-0095-9.

Sauerland, Uli. 1998. The meaning of chains: MIT PhD dissertation.

Sichel, Ivy. 2014. Resumptive pronouns and competition. Linguistic Inquiry 45. 655-693. doi:10.1162/ling_a_00169.

Steedman, Mark. 2011. Taking Scope: The Natural Semantics of Quantifiers. Cambridge, MA: MIT Press.

Szabó, Zoltán Gendler. 2010. Specific, yet opaque. In Amsterdam Colloquium, vol. 17, 32-41. doi:10.1007/978-3-642-14287-1_4.

Szabó, Zoltán Gendler. 2011. Bare quantifiers. Philosophical Review 120(2). 247-283. doi:10.1215/00318108-2010-029.

Ido Benbaji

32 Vassar Street

MIT Department of Linguistics and Philosophy

Cambridge, MA 02139

ibenbaji@mit.edu 